\title{
Militärkritik muss Fundamentalkritik sein, um kritisch zu bleiben
}

\author{
Michael Berndt*
}

\begin{abstract}
During the East-West conflict, the common focus of peace research was the possibility of an all destructing atomic war in Europe. On this common ground, a fundamental opposition against the military inspired by critical theory was able to become an integral part of peace research. With the end of the Cold War, especially the fundamental critique got out of fashion. One reason is that there was no advancement in this approach. But the adoption of neo-Gramscian and critical constructivist assumptions could contribute to a revitalization of the fundamental military critique as a critique of common assumptions about necessary violence.
\end{abstract}

Keywords: fundamental criticism, violence critique, military critique, Neo-Gramscianism, Critical Theory Fundamentalkritik, Gewaltkritik, Militärkritik, Neo-Gramscianismus, Kritische Theorie

\section{Einleitung ${ }^{1}$}

$\mathrm{E}$ in Blick auf die etablierte Friedensforschung zeigt: Militär und militärische Gewalt stehen nicht mehr grundsätzlich in der Kritik. Sie werden höchstens noch daraufhin befragt, ob sie für diesen oder jenen Zweck geeignet und legitim oder ungeeignet und illegitim erscheinen (Fischer/Sahm 2005: 62, Bake 2011: 211). Nun bedeutet dies nicht, dass Militär als Institution und der Einsatz militärischer Gewalt überhaupt nicht mehr kritisiert würden. Im Umfeld der Friedensbewegung finden sich etliche Arbeiten, die kontinuierlich die bundesdeutsche Militärpolitik (z.B.: Brehm/ Koch/Ruf/Strutynski 2012), die Militärpolitik der Europäischen Union (z.B. IMI 2009) und der NATO (z.B. Strutynski 2010, Wagner/Marischka/Seifert 2009) kritisieren. Die etablierte Friedensforschung nimmt sie jedoch nicht wahr. ${ }^{2}$ Diese Ignoranz mag mit einer „generellen Krise normativer Theorien und dem Siegeszug von Rationalismus und Empirismus in den Sozialwissenschaften [...] zusammenhängen“ (Fischer/Sahm 2005: 69). Dazu gehört auch ein anderes, distanzierteres Verhältnis jüngerer Friedensforscher und Friedensforscherinnen zur Normativität (ebenda: 69-70). Dieses drücke sich, wie Julika Bake (2011: 211) es formuliert, in einem „Rückgang politisch-ideologisch motivierter Forschung und einer Zunahme empirisch-analytischer Arbeiten“ aus.

Vielleicht haben die Ignoranz und die Abkehr von politischnormativ orientierten Ansätzen aber auch mit einem Problem zu tun, das Anhänger einer fundamentalkritischen Friedensforschung ernst nehmen müssen: ihren Mangel an theoretischer Weiterentwicklung. Nun ist fundamentale Militärkritik kein Selbstzweck. Vielmehr geht es um einen praktischen Auftrag, der sich aus dem normativen Anspruch ergibt, einen wissenschaftlichen Beitrag zu leisten, um Krieg und Gewalt als Formen des Konfliktaustrags aus dem gesellschaftlichen

* Dr. habil. Michael Berndt, Studienrat mit den Fächern Politik und Wirtschaft und Musik an einem nordhessischen Gymnasium und Privatdozent für Politikwissenschaft am Fachbereich Sozialwissenschaften der Universität Osnabrück.

1 Auf diesem Wege möchte ich Sabine Jaberg für viele wertvolle Kommentare danken.

2 Darauf weist auch hin, dass entsprechende Arbeiten in Überblickswerken zur Friedens- und Konfliktforschung der letzten Jahre (z.B. Jahn/Fischer/ Sahm 2005, Schlotter/Wisotzki 2011) nicht zitiert werden.
Verkehr zu entfernen. Dies vermag fundamentale Militärkritik aber nur, wenn sie auch in der wissenschaftlichen Diskussion präsent ist. Wie lässt sich aber fundamentale Militär- und Gewaltkritik so revitalisieren, dass sie auch in der wissenschaftlichen Diskussion wieder präsenter wird?

Zur Beantwortung dieser Frage wird in einem ersten Schritt der ursprüngliche Anspruch fundamentaler Militär- und Gewaltkritik sowie ihre wissenschaftlich theoretische Verankerung aufgezeigt. Der zweite Schritt verdeutlicht, wie und warum fundamentalkritische Ansätze zu Zeiten des Ost-West-Konflikts in größere wissenschaftliche und politische Zusammenhänge eingebunden waren. Der dritte Schritt diskutiert die weltpolitischen und friedenswissenschaftlichen Veränderungen, die zur Marginalisierung fundamentalkritischer Positionen führten. Da der Autor jedoch von ihrer Notwendigkeit überzeugt ist, werden im vierten Schritt Ansatzpunkte in neueren Theoriediskussionen identifiziert. Ein Fazit fasst die Argumentationskette zusammen.

\section{Anspruch und wissenschaftlich-theoretische Fundierung früherer fundamentaler Militärkritik}

Zu Beginn der 1970er Jahre trat nach Dieter Senghaas die Kritische Friedensforschung mit „drei analytischen Stoßrichtungen“ an. Dazu gehöre „erstens die empirische (im Sinne einer theoretisch angeleiteten empirischen Sozialforschung), zweitens die kritische (im Sinne von Ideologiekritik und Kritik politischer Ökonomie) und drittens die konstruktive (im Sinne der Entwicklung von Praxeologie und konkreten Handlungsstrategien zur Realisierung neuer Ziele und Aktionsprogramme)“ (Herv. i.O.) (Senghaas 1971: 18). Die Fundamentalkritik an allem Militärischen fügt sich in den zweiten genannten Strang. In diesem Sinne richtet sich „die kritische Spitze der Friedensforschung [...], gegen gesellschaftliche Strukturen und sozialpsychologische Bewusstseinsinhalte, die kollektive Aggressivität gerade auch innenpolitischer Art nicht nur vorbereiten, und schließlich ermöglichen, sondern geradezu fördern und die Kriegsbereitschaft wach halten. Friedensforschung sucht also die Ursache vor allem kriegerischer 
Konflikte und manifester oder latenter Gewaltstrukturen im innenpolitischen Bereich aufzudecken und auf sie praktisch im Sinne ihrer Beseitigung - einzuwirken“ (Senghaas 1973: 1516). Sie strebt "ganz dezidiert die Abschaffung von Kriegen als Mittel der Politik an“ (ebenda: 15). Das erfordere jedoch eine tiefgreifende „Veränderung der gesellschaftlichen Voraussetzungen“ (ebenda: 17), die sich auch auf den Wirtschaftssektor und das politische System erstrecke.

Letztlich geht es der Fundamentalkritik also um die Abschaffung der „gewaltgegründeten Macht von Menschen über Menschen“ (Krippendorff 1986: 11). Damit bewegt sie sich unverkennbar in der Nähe zu Max Horkheimers „Idee einer künftigen Gesellschaft als Gemeinschaft freier Menschen“ (Horkheimer 2003: 234). Diese wird jedoch nicht in das Reich abstrakter Utopien verwiesen, sondern von dieser unterscheide sie sich „durch den Nachweis ihrer realen Möglichkeit beim heutigen Stand der Produktivkräfte“ (ebenda: 236). Dies eröffnet letztlich auch Realiserungschancen für soziale Gerechtigkeit, die Johan Galtung (1969) als positiven Frieden bezeichnet. Allerdings sieht die Fundamentalkritik die Idee nicht als Selbstläufer. Vielmehr geht sie davon aus, dass sie in der Praxis dicke Bretter bohren muss. Das schreckt sie aber nicht ab. Denn sie unterstellt, dass ohne konkrete Utopien die vorherrschenden Gewaltverhältnisse nicht geändert werden können (Senghaas 1973: 19).

Drücken sich darin nun der normative Kern und die Leitgedanken fundamentalkritischer Analysen aus, so haben sie doch auch einen empirischen Kern: Nach Ekkehart Krippendorff (1985) liegen die Ursachen der bestehenden Gewaltverhältnisse in der sich global ausbreitenden Herrschaftsform des Staats auf Basis kapitalistischer Reproduktion. Damit wäre die erste Dimension kritischer Theorie, nämlich die Kritik politischer Ökonomie mit ihrem Fokus auf materiellen Interessen(lagen), angesprochen. In der auf ihr aufbauenden zweiten Dimension - der Ideologiekritik - widmet sie sich den Legitimationsstrukturen vorherrschender Politik und versucht, deren ideologische Prägung herauszuarbeiten.

Der wissenschaftliche Beitrag bestand und besteht in normativ motivierter, theoretisch angeleiteter empirischer Untersuchungen folgender Fragen: Worin liegen die konkreten Ursachen der Gewaltverhältnisse? Wie sehen deren konkrete aktuelle Ausprägungsformen aus? Was steht ihrer Überwindung entgegen? Wie kann diese vielleicht doch herbeigeführt werden? Damit will Friedensforschung Aufklärung in praktischer Absicht leisten. Allerdings scheidet für fundamentalkritische Ansätze (militärische) Gewalt aus dem zulässigen Handlungsrepertoire - konsequent zu Ende gedacht - kategorisch aus, beinhaltet sie doch genau jene Gewaltförmigkeit, die sie zu bekämpfen beabsichtigt. ${ }^{3}$

3 Der Sachverhalt, dass in den 1970er und 1980er Jahren, sofern es um so genannte Befreiungsbewegungen in der Dritten Welt ging, die Gewaltablehnung lange nicht so konsequent war wie z.B. gegenüber den USA, soll hier nicht unterschlagen werden. Nach dem Ende des realexistierenden Sozialismus und somit einer vermeintlichen Alternative kann die Gewaltkritik endlich logisch und konsequent zu Ende gedacht werden.

\section{Militär- und Gewaltkritik zu Zeiten des Ost- West-Konflikts}

Angesichts der Gefahr eines verheerenden Nuklearkriegs konzentrierte sich zu Zeiten des Ost-West-Konflikts nahezu die gesamte Disziplin der Friedensforschung über ihre weltanschauliche und wissenschaftstheoretische Gräben hinweg auf die Kritik an staatlich organisierter militärischer Gewalt. Im Mittelpunkt stand dabei die Militärstrategiekritik ${ }^{4}$. D.h. es wurden Militärstrategien und auf ihr basierende Waffentechnologien kritisiert, die beinhalteten, dass im Falle eines Atomkriegs das, was eigentlich durch militärische Verteidigung hätte geschützt werden sollen, zerstört worden wäre (siehe z.B. Bielfeldt/ Schlotter 1980; Bredow/Thielen 1974). In diesem Kontext gab es einen gemeinsamen (politischen) Bezugspunkt aller Friedensforschung, nämlich mit wissenschaftlichen Mitteln einen Beitrag zur Verhinderung eines Atomkriegs zu leisten. Mithin besaß sie auch einen normativen Minimalkonsens des Inhalts: ,Atomkrieg darf nicht sein'.

Fundamentalkritische Positionen befanden sich hier in der Minderheit. Sie waren aber mitnichten marginalisiert, sondern eingebettet in den größeren Kreis der Friedensforschung. Ihre Thesen wurden wahrgenommen und diskutiert. ${ }^{5}$ Als Militärpolitikkritik ging Fundamentalkritik aber über reine Strategiekritik hinaus. Mit Krippendorffs o.g. These vom Zusammenhang zwischen modernem bürgerlich-kapitalistischen Staat und Krieg rückte darüber hinaus die gesamte Vergesellschaftungsform ins Visier. Gehören der moderne kapitalistische bürgerliche Staat und Militär bzw. Krieg untrennbar zusammen, dann kommt letztlich nur ein ebenso radikaler wie komplexer Lösungsweg in Frage: Abschaffung des Militärs sowie Veränderung staatlicher Herrschaft und Eingriffe in ökonomische Reproduktionsbedingungen. Diesem Weg folgten nachvollziehbarerweise nicht alle, mit denen bei der Strategiekritik noch Einvernehmen herrschte. Doch auch die Verfechter eines moderateren Ansatzes setzten sich mit den Inhalten der Fundamentalkritik auseinander. Dies lag durchaus nahe, weil sie sich doch zumindest in Teilen auf ein gemeinsames Anliegen, nämlich die Vermeidung des Nuklearkriegs, bezog (siehe z.B. Czempiel 1986: 26; Münkler 1987; Jahn 1988).

\section{Krise der Fundamentalkritik nach Ende des Ost-West-Konflikts}

Das Ende des Kalten Kriegs wurde Anfang der 1990er Jahre mit einer Reihe ,heißer Kriege' eingeläutet: Dazu zählten der zweite Golfkrieg ebenso wie die Staatszerfallskriege im ehemaligen

4 Militärkritik und gerade auch fundamentale Militärkritik besaß noch viele weitere Dimensionen. Zu nennen sind hier u.a. die Kritik an der auf Befehl und Gehorsam basierenden Organisation des Militärs, die Kritik des Einzugs militärischer Sprache in andere gesellschaftliche Bereiche (z.B. Sport) und die Kritik der (Re-)Konstruktion von Geschlechterverhältnissen. Siehe dazu die Beiträge in der Zeitschrift „antimilitarismus information“, der Schriftenreihe der Arbeitsgemeinschaft Friedens- und Konfliktforschung und des "Anti-Weißbuches" (Studiengruppe Militärpolitik 1974). Aktuell sei auch auf den Sammelband „Banal Militarism“ (Thomas/Virchow 2006) verwiesen, der aber von der etablierten Friedensforschung auch kaum wahrgenommen wurde. Im Sammelband von Schlotter/Wisotzki (2011) wird er kein einziges Mal zitiert.

5 Dies zeigen z.B. die Beiträge in dem Sammelband Haftendorn (1975). 
Jugoslawien. Damit änderten sich die Rahmenbedingungen, die bis dahin die Einbindung der Fundamentalkritik in die Friedensforschung begünstigten.

Zunächst standen alle theoretischen Herangehensweisen auch in der Friedensforschung unter Rechtfertigungszwang, hatten sie doch das Ende des Ost-West-Konflikts nicht antizipiert (Holm/Sorensen 1994: 1). Dies führte zu einer Konjunktur bisher weniger beachteter Ansätze; insbesondere die Konstruktivismus- und die Postmoderne-Diskussionen erlebten einen Aufschwung. Während es den moderateren Ansätzen in der Friedensforschung gelang, sich mit den neuen Diskursen zu arrangieren, ihre Herangehensweisen aufzunehmen und für die eigene theoretische Weiterentwicklung zu nutzen, tat sich die Fundamentalkritik damit weitaus schwerer, erschien ihr doch gerade der zweite Golfkrieg als Bestätigung ihres bisherigen politökonomischen Paradigmas. Da ihre Anhänger hier die klassische Rohstoff-Interessenpolitik am Werke sahen (siehe z.B. Ruf 1991), lehnten sie konstruktivistische Ansätze als zu wenig materialistisch ab. Teilweise erschienen diese neueren Theorien gar als Ideologie, die es zu kritisieren galt, statt sie als Angebote für die eigene theoretische Weiterentwicklung zu begreifen. So erging es im deutschsprachigen friedenswissenschaftlichen Raum sogar Ansätzen, die von der Kritik der politischen Ökonomie gar nicht so weit entfernt waren. Die Neo-Gramsci-Diskussion fand zwar im Politikfeld der internationalen politischen Ökonomie einige Anhänger (siehe z.B. die Beiträge in: Opratko/Prausmüller 2011), für die Gewaltkritik wurde sie aber kaum herangezogen. Die Fundamentalkritik blieb so im theoretischen Bereich auf dem Stand vor Ende des Ost-West-Konflikts. Nun hatten sich aber eben die Rahmenbedingungen geändert: Die Wahrscheinlichkeit eines Atomkriegs in Europa nahm ab, entsprechend relativierte sich die Kritik an den Gefahren militärischer Eskalationsstrategien. Ein nahezu verschwundenes Problem brauchte zu seiner Lösung keinen ansatzübergreifenden Konsens mehr.

In der Folge setzten die weniger radikalen Strömungen fundamentalkritische Positionen mit der These unter Druck, Gewalt könne notwendig sein, um vorgängige Gewalt zu beenden. Mit dem so genannten Pazifismus-Bellizismus-Streit (siehe: Wasmuht 1998, Krell 1998) kam es zum Bruch, in dessen Folge die Fundamentalkritik in der etablierten Friedensforschung an Bedeutung verlor. Den Hintergrund dieser Diskussion bildeten vor allem die jugoslawischen Zerfallskriege. Nun avancierte militärische Gewalt auch für Teile der Friedensforschung zum gegebenenfalls notwendigen Mittel - zumal dann, wenn sie völkerrechtlich unproblematisch oder zumindest legitimierbar schien. Unter der Bedingung, dass Militäreinsätze nicht mehr zu einem alles vernichtenden Krieg zu eskalieren drohen, relativiert sich auch die Bedeutung der Gewaltkritik. Nunmehr scheinen humanitäre Ziele und Werte den Gewaltmitteleinsatz rechtfertigen zu können.

Das Zusammenwirken beider Faktoren - veränderte weltpolitische Rahmenbedingungen und theoretische Stagnation - hat zum Bedeutungsverlust der Fundamentalkritik geführt, zumal sich mit Dieter Senghaas oder auch Egbert Jahn exponierte Vertreter der ersten Stunde von ihr abwandten. Geblieben ist auf theoretischer Ebene eine Staats-, Militär- und Gewaltkritik, über die Jahn schon 1988 - als eigene Verabschiedung von der Kritischen Friedensforschung - urteilte: „Liegt das Übel in irgendeinem ,System', so hilft nur der radikale, , an die Wurzel gehende' Umsturz, geleitet vom guten Willen, sich nicht mit den vorhandenen Gewaltverhältnissen abzufinden, genügen nicht die im Geschichtsprozess angelegten und erkennbaren Veränderungskräfte." (Jahn 1988: 105)

Statt die Fundamentalkritik und den normativen Anspruch aufzugeben, wäre zu untersuchen, „wie es einem historischkonstituierten Bürgertum immer wieder gelingt, die antagonistischen Beziehungen in nichtantagonistische Kooperationsformen zu verwandeln" (Röttger 1998: 8) und damit Gewaltverhältnisse zu reproduzieren. Um dies theoriegeleitet empirisch herausarbeiten zu können, ist eine theoretische Weiterentwicklung der Fundamentalkritik nötig. Andernfalls verengte sie sich auf die Herausarbeitung (singulärer) systemimmanenter materieller Interessen(lagen). ${ }^{6}$ Besitzen diese zwar auch weiterhin ihren Erklärungswert, so greift eine derartige Reduktion aber zu kurz. In diesem Sinne stellt Mario Candeias (2003: 61) fest, „dass kein Konflikt allein auf ökonomische Ursachen zurückzuführen ist. Es geht immer um die spezifische Artikulation ökonomischer mit strategischen Interessen, Menschenrechtsdiskursen, internationalem Recht und nicht zuletzt innenpolitischen Verhältnissen. Es geht immer auch ums Ganze, die Verteidigung einer bestimmten Produktions- und Lebensweise".

Die analytische Verkürzung militärischer Gewalt im Kosovo, in Afghanistan, im Irak, in Libyen oder Mali auf ökonomische Interessen wird ihrer Komplexität nicht gerecht. Derartige Erklärungen helfen auch wenig dabei, die Frage zu beantworten, warum sich die Ablehnung internationaler Bundeswehreinsätze durch die Mehrheit der deutschen Bevölkerung nicht bei Bundestagswahlen niederschlägt. Die These, die Bevölkerung setze andere Prioritäten und nehme deshalb Auslandseinsätze einfach hin, erklärt wohl nur einen Teil des Befunds. Der andere Teil besteht m.E. darin, dass sowohl über die Konfliktkonstruktion als auch die Ziele der Interventionen weitgehend Konsens herrscht und letztlich nur die Mittelfrage wirklich umstritten ist. Das Problem dabei besteht allerdings darin, dass Zieldefinition und Konfliktkonstruktion auch die Wahl der Mittel entscheidend beeinflussen. Monokausale Erklärungsmuster helfen dabei kaum weiter. Stattdessen könnte die Verarbeitung ,neuerer' Theoriediskussionen der Fundamentalkritik wichtige Impulse setzen.

\section{Neuere Theorieentwicklungen und Revitalisierung fundamentaler Militär- und Gewaltkritik}

Wird sich also nicht vorschnell von fundamentaler Gewaltund Militärkritik verabschiedet, dann springen zunächst die neuen politischen Sprachregelungen zu Militäreinsätzen ins Auge, die sich auch in der Friedensforschung finden. Heutzutage wird nicht mehr Krieg geführt, sondern Frieden geschaffen. Es geht nicht mehr um die Durchsetzung eigener materi-

6 Siehe dazu die genannten Arbeiten aus dem Kreis des Bundesausschusses Friedensratschlag und der Informationsstelle Militarisierung. 
eller Interessen oder Weltordnungsvorstellungen, sondern um human security, Menschenrechte und Demokratie, nicht mehr um das staateneigene Recht zum Krieg, sondern um responsibility to protect. Statt von Herrschaft ist von governance die Rede.

Wenn diese Sprachregelungen nicht einfach übernommen, sondern hinterfragt werden sollen, dann bleibt ein fundamentaler Ansatz notwendig. Andernfalls ginge es nur noch um die Frage, unter welchen Bedingungen Gewalt notwendig ist. Fundamentalkritik muss sich also der Aufgabe stellen aufzuzeigen, wie für (militärische) Gewalt Akzeptanz geschaffen wird. Dabei genügt es nicht, lediglich dezidierte Falschinformationen nachzuweisen (siehe: Cárdenas/Nachtigall/Nau/Wette 2013). Vielmehr gilt es grundlegende Denkmuster zu hinterfragen, die Gewalt unter bestimmten Bedingungen als notwendig erscheinen lassen. In den Fokus der Analyse rückten damit Medien, politische Erklärungen, Bildungsangebote, aber auch wissenschaftliche Erklärungsversuche. Dabei geht es um die Dekonstruktion sogenannter „intersubjective meanings” (Cox 1986: 218), also gesellschaftlich unstrittiger Bedeutungen. Eine fundamental kritische Argumentation muss genau dieses Konsensuale als Rahmenbedingung für die Akzeptanz militärischer Gewalt grundsätzlich hinterfragen. Drei miteinander zusammenhängende Dimensionen gilt es zu beachten (siehe Berndt 2007: 63ff.). Sie müssen allesamt durchleuchtet bzw. dekonstruiert werden, um der fortwährenden Begründung militärischer Gewalt den Boden zu entziehen:

1. die Konstruktion von Weltordnungen (einschließlich der Konstruktion des zu Schützenden und des Bedrohenden),

2. die Konstruktion notwendiger Gewalt und

3. die Konstruktion von Sicherheit und Unsicherheit.

\subsection{Dekonstruktion von Weltordnungsvorstellungen}

Wenngleich auf der einen Seite weiter von materiellen Interessenlagen und ihrem Einfluss auf politische Entscheidungen ausgegangen wird, gesellt sich auf der anderen Seite das Problem hinzu, wie sich Interessen Akzeptanz verschaffen. Dabei kommt der Antwort auf die Frage entscheidende Bedeutung zu, wie die einschlägigen Diskurse gesellschaftliche Verhältnisse so konstruieren, dass (militärische) Gewalt als notwendiges Mittel erscheint. Das bedeutet nicht, dass die militärische Option immer explizit propagiert werden müsste. Entscheidend ist der implizite Bereich, in dem Gewalt nicht ausgeschlossen wird. Hier stellt sich zunächst die Frage nach vorherrschenden Weltordnungsvorstellungen und wie innerhalb dieser Vorstellungen das ,Inside' und das ,Outside' (Walker 1993), das ,We' und das ,Other' charakterisiert werden. In diesem Sinne stellt Rob Walker (1995: 321) fest: „Inside the particular, aspirations for the universal can be pursued within a secure and authoritative order, although it is best not to think about the behaviours required at the margins of society where violence may be necessary to sustain the claims of righteousness within. Outside, there are only other particulars, an absence of a cohesive community, a pure anarchy according to some, a decentralized society of states according to others, but a realm, certainly, of difference, competition, insecurity, domination and conflict: a realm in which others can be turned into Other, and the Other may be subjected to the familiar practices of projection, negation, orientalism and obliteration."

Denn in Abhängigkeit von der Konstruktion des ,Anderen‘ erscheint es mehr oder weniger gerechtfertigt, ihm gegenüber Gewalt anzuwenden. Und diese Gewalt gilt erst recht dann als notwendig, wenn das ,Andere' die als schützenswert deklarierte Ordnung bedroht. Gewaltkritik ist damit Kritik solcher Weltordnungsvorstellungen, denen militärische Gewalt immanent ist - und zwar sowohl als bedrohende Gewalt als auch als (vermeintlich) schützende Gewalt.

\subsection{Dekonstruktion notwendig erscheinender Gewalt}

Christoph Weller (2003: 494) stellt fest, dass „[j]ede Gesellschaft [...] ihren Konsens über Formen von Gewalt [besitzt], die eindeutig illegitim bzw. legitim sind“ (Herv. i.O.). Und er fährt fort: „Gewalt produziert Legitimation für Gegen-Gewalt, oder friedenstheoretisch präzisiert: Die gesellschaftliche Bewertung bestimmter Gewaltformen als illegitim verschafft der entsprechenden Gegengewalt gesellschaftliche Legitimation." (ebenda). Aus fundamentalkritischer Perspektive geht es jedoch nicht darum, sich wie Weller „auf der Grundlage friedensethischer Argumente um eine Positionsbestimmung zu genau jener Grenzziehung zwischen legitimer und illegitimer Gewalt [zu] bemühen" (ebenda: 501) und damit bestimmte Formen von Gewalt zu legitimieren. Im Gegenteil besteht ihr Anliegen darin, den Konsens über legitime Gewalt aufzubrechen.

Genau hier ist dann zu analysieren, wem oder was gegenüber und in welchen Kontexten Gewalt als allseits anerkannt gilt. Entsprechend formuliert Bradley Klein (1988: 136) schon 1988 aus neo-gramscianischer Perspektive: „In this sense, popular representations of violence and of the ,enemy' against whom violence is to be legitimately deployed become significant artefacts insofar as they construct as plausible a distinct range of identities and render others unavailable or implausible. To study strategic culture is to study the cultural hegemony of organized state violence." Gewaltkritik in diesem Sinne ist Kritik einer Konstruktion von Feinden, die impliziert, dass gegen sie nur Gewalt hift.

\subsection{Dekonstruktion des Sicherheitsdiskurses}

Gleiches gilt im Prinzip für die Securitization-These (siehe dazu: Wæver 1997), die die Fundamentalkritik bislang kaum rezipiert hat, erscheint sie ihr doch zu konstruktivistisch. Aber aus dieser These lässt sich auch ableiten, dass bestimmte Entwicklungen genau deshalb als sicherheitsrelevant definiert werden, um „a special right to use the means necessary to block this development" (ebenda: 22) zu beanspruchen. Dieses fundamentalkritische Potenzial gilt es zu nutzen. 
Christopher Daase (2010) identifiziert bei der Sicherheitsdiskussion ein Problem: Ausufernde gesellschaftliche Sicherheitsbedürfnisse würden „nicht nur zu institutionellen Veränderungen und rechtlichen Verwerfungen in der Sicherheitspolitik führ[en], sondern generell staatliche Sicherheitsorgane und internationale Sicherheitsinstitutionen zu überfordern beginn[en]" (ebenda: 16). Einer fundamentalkritischen Perspektive drängte sich demgegenüber eine andere Frage auf: (Re-)Produziert nicht die fortwährende Anrufung von Sicherheit die Rechtfertigung militärischer Gewalt zur Sicherheitsvorsorge? Gewaltkritik ist somit eine Kritik von gewaltlegitimierenden Sicherheitsdiskursen.

\section{Fazit}

Die Kritische Friedensforschung trat zu Beginn der 1970er Jahre mit der Annahme an, dass Gewalt als Mittel der Politik nicht naturgegeben, sondern menschengeschaffen ist, mithin eliminiert werden kann. Dies führte sie zur Kritik an den gesellschaftlichen Verhältnissen. Ihre fundamentale Militär- und Gewaltkritik ist normativ. Als wissenschaftliche Veranstaltung muss sie aber auch auf dem aktuellen Stand der Theoriedebatten empirisch arbeiten können. Dies ist ihr jedoch spätestens seit Ende des Ost-West-Konflikts nicht mehr gelungen. Entsprechend fördert ihre empirische Arbeit immer nur Variationen des ewig gleichen Befunds zutage, der materielle ökonomische Interessen für militärische Gewalt verantwortlich macht. Nicht, dass dieser Aspekt irrelevant geworden wäre. Unter Hinzuziehung neuerer Theoriediskussionen ist es aber möglich, die kritischen Fragen zu erweitern, um gerade die Mechanismen der Akzeptanzreproduktion für Gewaltverhältnisse in den Blick zu bekommen. Mit diesem Fokus ergeben sich für die Fundamentalkritik Möglichkeiten, ihrerseits auf aktuelle Theoriediskussionen einzuwirken. Zwar bestehen zwischen fundamentalkritischen Positionen und konstruktivistischen Herangehensweisen, wie sie z.B. Weller und Daase vertreten, entscheidende Unterschiede, doch ergeben sich auch Bezugspunkte für eine beidseitig fruchtbare Theoriediskussion. Solche theoretischen Impulse wären aus der Perspektive einer arg geschrumpften Fundamentalkritik aufzunehmen, um wissenschaftlich wieder an Attraktivität zu gewinnen. Dass dabei einerseits eine fundamentale Gewaltkritik auch einige immanente Probleme im Theoriebereich zu klären hat, soll hier nicht verschwiegen werden. So bedarf es u.a. einer Präzisierung der Antwort auf die Frage, wie sich divergierende materielle Interessen(lagen) zu politischen Konsensen insbesondere bezüglich Gewalt verhalten. Andererseits lassen sich aber mit den skizzierten Dekonstruktionsaufgaben jetzt schon diverse Phänomene auf Mikro-, Meso- und Makroebene in fundamentalkritischen Augenschein nehmen. Gerade der gesamte Medien- und Bildungsbereich liefert reichhaltiges Material für normativ motivierte, theoretisch angeleitete empirische Untersuchungen. Dabei ergeben sich stets Verbindungslinien zu anderen - konstruktivistischen oder auch neo-gramscianischen - Ansätzen. Präsenz im Theoriebereich bleibt also unverzichtbar für jede wissenschaftliche Weiterentwicklung. Dabei muss auch die normative Fundierung funda- mentaler Gewaltkritik immer wieder zur Diskussion, aber eben nicht in Frage stehen.

\section{Literatur}

Bake, Julika 2011: Militär und Gesellschaft in Deutschland, in: Schlotter, Peter/Wisotzki, Simone (Hrsg.): Friedens- und Konfliktforschung (Schriftenreihe der Arbeitsgemeinschaft für Friedens- und Konfliktforschung: 35); Baden-Baden; S. 183222.

Berndt, Michael 2007: Die „Neue Europäische Sicherheitsarchitektur“. Sicherheit in, für und vor Europa?; Wiesbaden.

Bielfeldt, Carola/Schlotter, Peter 1980: Die militärische Sicherheitspolitik der Bundesrepublik Deutschland. Einführung und Kritik; Frankfurt a.M./ New York.

Bredow, Wilfried von/Thielen, Joseph H. Helmut 1974: Kritik der Sicherheitspolitik der BRD, in: Studiengruppe Militärpolitik; Ein Anti-Weißbuch. Materialien für eine alternative Militärpolitik; Reinbek; S. 15-45.

Brehm, Maybritt/Koch, Christian/Ruf, Werner/Strutynski, Peter 2012: Armee im Einsatz. 20 Jahre Auslandseinsätze der Bundeswehr; Hamburg.

Candeias, Mario 2003: Terror und Krieg. Widersprüche neoliberaler Globalisierung, in: Forum Wissenschaft (20: 3); S. 59-63.

Cárdenas Alfonso, Maria/Nachtigall, Andrea/Nau, Johannes/Wette, Wolfram 2013: Nichts als die Wahrheit? Über die Kreativität der Unwahrheit im Kontext von Krieg und Gewalt (Wissenschaft und Frieden/Dossier: 72); Bonn.

Cox, Robert W. 1986: Social forces, states and world orders. Beyond international relations theory, in: Keohane, Robert O. (Hrsg.): Neorealism and its Critics; New York; S. 204-254.

Czempiel, Ernst-Otto 1986: Friedensstrategien. Systemwandel durch Internationale Organisationen, Demokratisierung und Wirtschaft; Paderborn/München/Wien.

Daase, Christopher 2010: Wandel der Sicherheitskultur, in: Aus Politik und Zeitgeschichte (60: 50); S. 9-16.

Fischer, Sabine Sahm, Astrid 2005: Friedensforschung und Normativität: Positionen der jüngeren Generation, in: Jahn, Egbert/Fischer, Sabine/Sahm, Astrid (Hrsg.): Die Zukunft des Friedens, Bd 2: Die Friedens- und Konfliktforschung aus der Perspektive der jüngeren Generation; Wiesbaden; S. 49-72.

Galtung, Johan 1969: Violence, peace and peace research, in: Journal of Peace Research (6: 2); S. 167-191.

Haftendorn, Helga 1975 (Hrsg.): Theorie der Internationalen Politik - Gegenstand und Methode der Internationalen Beziehungen; Hamburg.

Holm, Hans-Henrik/Sorensen, Georg 1994: Introduction: What has Changed?, in: Holm, Hans-Henrik/Sorensen, Georg (Hrsg.): Whose World Order? Uneven Globalization and the End of the Cold War; Boulder/San Francisco/Oxford; S. 1-17. 
Horkheimer, Max 2003: Traditionelle und kritische Theorie, in: Horkheimer, Max: Traditionelle und kritische Theorie. Fünf Aufsätze; Frankfurt a.M. (5. Aufl.); S. 205-259.

IMI (Informationsstelle Militarisierung) 2009 (Hrsg.): Militärmacht EUropa: Eine Zwischenbilanz; Tübingen.

Jahn, Egbert K. 1988: Von der internationalen Friedensforschung zur nationalen Sicherheitsforschung?, in: Moltmann, Bernhard (Hrsg.): Perspektiven der Friedensforschung (Schriftenreihe der Arbeitsgemeinschaft für Friedens- und Konfliktforschung : XV); Baden-Baden; S. 85-110.

Jahn, Egbert/Fischer, Sabine/Sahm, Astrid 2005 (Hrsg.): Die Zukunft des Friedens. Bd. 2: Die Friedens- und Konfliktforschung aus der Perspektive der jüngeren Generation; Wiesbaden.

Klein, Bradley S. 1988: Hegemony and strategic culture. American power projection and alliance defence politics, in: Review of International Studies (2: 14); S. 133-148.

Krell, Gert 1998: Konfliktfähiger Pazifismus. Ein Kommentar, in: Grundmann, Martin/Hummel, Hartwig (Hrsg.): Militär und Politik. Ende der Eindeutigkeiten? Zum Wandel institutionalisierter Gewalt (Schriftenreihe der Arbeitsgemeinschaft für Friedens- und Konfliktforschung: XXV); Baden-Baden; S. 41-50.

Krippendorff, Ekkehart 1985: Staat und Krieg. Die historische Logik politischer Unvernunft; Frankfurt a.M.

Krippendorff, Ekkehart 1986: Internationale Politik. Geschichte und Theorie; Frankfurt a.M./New York.

Münkler, Herfried 1987: Staat, Krieg und Frieden: Die verwechselte Wechselbeziehung. Eine Auseinandersetzung mit Ekkehart Krippendorff: Staat und Krieg. Die Historische Logik politischer Unvernunft, in: Hessische Stiftung Friedens- und Konfliktforschung (Hrsg.): Kriegsursachen (Friedensanalysen: 21); Frankfurt a.M.; S. 135-144.

Opratko, Benjamin/Prausmüller, Oliver 2011 (Hrsg.): Gramsci global: Neogramscianische Perspektiven in der Internationalen Politischen Ökonomie (Argument Sonderband: 310); Hamburg.

Röttger, Bernd 1998: Wiederbelebung eines toten Hundes. Grundlegung, Entwicklungen und Zurichtungen der Kritik der politischen Ökonomie - 150 Jahre nach dem „Kommunistischen Manifest“, in: Forum Wissenschaft (15: 3); S. 6-9.

Ruf, Werner 1991: Aussichten auf die neue Welt-Un-Ordnung, in: Ruf, Werner (Hrsg.): Vom Kalten Krieg zur heißen Ordnung. Der Golfkrieg: Hintergründe und Perspektiven; Münster; S. 83-96.

Sahm, Astrid/Sapper, Manfred/Weichsel, Volker 2002 (Hrsg.): Die Zukunft des Friedens. Eine Bilanz der Friedens- und Konfliktforschung; Wiesbaden.

Schlotter, Peter/Wisotzki, Simone 2011 (Hrsg.): Friedens- und Konfliktforschung (Schriftenreihe der Arbeitsgemeinschaft für Friedens- und Konfliktforschung : 35); Baden-Baden.
Senghaas, Dieter 1971: Editorisches Vorwort, in: Senghaas, Dieter (Hrsg.): Kritische Friedensforschung; Frankfurt a.M.; S. 7-21.

Senghaas, Dieter 1973: Einleitung zur ersten Auflage 1970, in: Senghaas, Dieter (Hrsg.): Friedensforschung und Gesellschaftskritik; Frankfurt a.M.; S. 10-22.

Strutynski, Peter 2010: NATO im Wandel. Das größe Militärbündnis der Geschichte auf der Suche nach einer neuen Strategie, in: Österreichisches Studienzentrum für Frieden und Konfliktlösung (Hrsg.): Demokratie im globalen Wandel. Eine Welt im demokratischen Aufbruch?. Friedensbericht 2010; Münster/Wien; S. 183-195.

Studiengruppe Militärpolitik 1974: Ein Anti-Weißbuch. Materialien für eine alternative Militärpolitik; Reinbek.

Thomas, Tanja/Virchow, Fabian 2006 (Hrsg.): Banal Militarism. Zur Veralltäglichung des Militärischen im Zivilen (Cultural Studies 13); Bielefeld.

Woever, Ole 1997: Securitization and desecuritization, in: Wæver, Ole: Concepts of security; Copenhagen; S. 211-256.

Wagner, Jürgen/Marischka, Christoph/Seifert, Andreas 2009 (Red.): Kein Frieden mit der NATO. Die NATO als Waffe des Westens (Informationsstelle Militarisierung (IMI)/DFG-VK (Hrsg.)); Tübingen.

Walker, R.B.J. (Rob) 1993: Inside/Outside. International relations as political theory; Cambridge.

Walker, R.B.J. (Rob) 1995: International relations and the concept of the political, in: Booth, Ken/Smith, Steve (Hrsg.): International Relations Theory Today; Pennsylvania; S. 306-327.

Wasmuht, Ulrike C. 1998: „Frieden schaffen, mit Waffen?“ Zur Uneindeutigkeit der Bellizismus-, Belli-Pazifismus- und Pazifismusdebatte, in: Grundmann, Martin/Hummel, Hartwig (Hrsg.): Militär und Politik. Ende der Eindeutigkeiten? Zum Wandel institutionalisierter Gewalt (Schriftenreihe der Arbeitsgemeinschaft für Friedens- und Konfliktforschung: XXV); Baden-Baden; S. 19-40.

Weller, Christoph 2003: Gewalt - politischer Begriff und friedenswissenschaftliche Konzepte. Eine Kritik der Gewaltfreiheit des Friedens, in: Calließ, Jörg/Weller, Christoph (Hrsg.): Friedenstheorie. Fragen - Ansätze - Möglichkeiten (Loccumer Protokolle: 31/03); Loccum; S. 481-508. 